Ciencias de la Salud

Articulo original

\title{
Efectos de aplicación de gel de doxiciclina y solución de clorhexidina en
} tratamientos bucales

Effects of Doxycycline gel application and chlorhexidine solution in oral treatments

Efeitos da aplicação do gel de doxiciclina e clorohexidina solução em tratamentos

orais

\section{Esp. Carina G. Orbea-Iturralde, Dra. Alicia C. Martínez-Martínez, Dra. Mariela C. Balseca-Ibarra gabriela.orbea@gmail.com, aliciacomartinez@ hotmail.com, marielabalsecai@yahoo.com, mbalseca@uce.edu.ec}

Universidad Central del Ecuador, Quito, Ecuador

Recibido: 30 de octubre de 2015

Aceptado: 13 de septiembre de 2016

\section{Resumen}

Objetivo: Evaluar y comparar los efectos clínicos de la aplicación de gel de doxiciclina al 20\% vs clorhexidina al $0.2 \%$ después de la terapia periodontal básica. Materiales y métodos: La presente investigación tuvo un estudio de campo, cuantitativo, cualitativo y bibliográfico; a boca dividida en el cual se seleccionó varios dientes diagnosticados con enfermedad periodontal crónica. Se realizó tratamiento periodontal básico que consiste en el raspado y alisado radicular, empleándose además dos tipos de sustancias de manera local: gel de doxiciclina al $20 \%$ en el sitio de la bolsa manteniéndose por un periodo de 7 días cubierto con cemento quirúrgico, y solución de clorhexidina al $0.2 \%$ en forma de irrigación, se utilizó una tabla de vaciado de datos con el fin de sistematizar la información en tablas y gráficas de estadística descriptiva, así como la realización de las pruebas de estadísticas con el paquete de datos SPSS versión 20. Resultados: En el análisis de los resultados se utilizó la prueba t de Student para establecer una comparación entre las dos técnicas. Los resultados demuestran que: para el índice de placa los resultados fueron estadísticamente significativos para los dos grupos. El nivel de inserción fue mayor luego del 
tratamiento. Al comparar las dos técnicas de tratamiento para el valor inicial y final del nivel de inserción, la prueba t para muestras emparejadas determinó para los dos grupos un valor $\mathrm{p}=0$, permitiendo concluir que si hay diferencias significativas en el nivel de inserción antes y después de tratamiento con las dos técnicas. En la profundidad de sondaje para las dos técnicas de tratamiento en estudio se evidenció disminución post tratamiento significativa, dado que $\mathrm{p}=0$. En cuanto al sangrado se registró una mayor eficacia para la doxicilina, que logró una disminución del 67\% inicial a 8,9\% post tratamiento. Conclusiones: Para todos los parámetros clínicos: profundidad de sondaje, nivel de inserción, placa, sangrado, hubo diferencias significativas antes y después del tratamiento empleando las dos técnicas, gel de doxiciclina $20 \%$ y clorhexidina $0.2 \%$, siendo el sangrado el más notable en su reducción con el uso de doxiciclina de $67 \%$ a 8,9\%.

Palabras Clave: enfermedad periodontal; doxiciclina; clorhexidina; periodontopatógenas.

\section{Abstract}

Objective: To evaluate and compare the clinical effects of the application of doxycycline gel $20 \%$ vs $0.2 \%$ chlorhexidine after basic periodontal therapy. Materials and methods: This research is a field study, quantitative, qualitative and literature; with chronic periodontal disease were selected. solution doxycycline gel $20 \%$ at the site of the bag being maintained for a period of seven days covered with surgical cement, and Basic periodontal treatment that involves scaling and root planing was performed, besides being used two types of substances locally $0.2 \%$ chlorhexidine as irrigation, Dump table data was used in order to systematize information in tables and graphs of descriptive statistics and conducting statistical tests with the data package SPSS version 20. Results: Student t test was used in the analysis of the results for comparison between the two techniques. The results show that: for plaque index results were statistically significant for both groups. Attachment level was higher after treatment. When comparing the two treatment techniques for the initial and final value of the level of insertion, the $t$ test for paired samples determined for the two groups $\mathrm{p}=0$ value, leading to the conclusion that if no significant differences in the level of insertion before and after treatment with both techniques. In probing depth for the two treatment techniques in post study it showed significant decrease treatment, since $\mathrm{p}=0$. In terms of bleeding increased efficiency for doxycycline, which achieved a $67 \%$ decrease was recorded initial 8, 9\% post treatment. Conclusions: For all clinical parameters: probing depth, attachment level, plaque, bleeding, significant differences before and after treatment using both techniques, doxycycline gel $20 \%$ and 
chlorhexidine $0.2 \%$, the bleeding most notably in reduction with the use of doxycycline from $67 \%$ to $8.9 \%$.

Keywords: Periodontal disease; doxycycline; chlorhexidine; periodontopathogenic.

\section{Resumo}

Objetivo: Avaliar e comparar os efeitos clínicos da aplicação do gel de doxiciclina $20 \%$ vs $0,2 \%$ clorexidina após a terapia periodontal básica. Materiais e Métodos: Esta pesquisa é um estudo de campo, quantitativa, qualitativa e literatura; com doença periodontal crônica foram selecionados. solução de gel de doxiciclina $20 \%$ no local da bolsa a ser mantido por um período de sete dias coberto com cimento cirúrgico, e tratamento periodontal básico que envolve raspagem e alisamento radicular foi realizada, além de serem utilizados dois tipos de substâncias localmente $0,2 \%$ de clorexidina como a irrigação, dados da tabela despejo foi usada para sistematizar informações em tabelas e gráficos de estatísticas descritivas e realização de testes estatísticos com o pacote de dados SPSS versão 20. Resultados: Teste t de Student foi utilizado para a análise dos resultados de comparação entre as duas técnicas. Os resultados mostram que: para os resultados do índice de placa foram estatisticamente significativas para ambos os grupos. nível de inserção foi maior após o tratamento .. Ao comparar as duas técnicas de tratamento para o valor inicial e final do nível de inserção, o teste t para amostras pareadas determinados para os dois grupos $\mathrm{p}=0$ valor, levando à conclusão de que, se não significativa as diferenças no nível de inserção antes e depois do tratamento com as duas técnicas. Em profundidade de sondagem para as duas técnicas de tratamento em estudos de pós mostrou tratamento significativa diminuição, uma vez que $p=0$. Em termos de sangramento maior eficiência de doxiciclina, que alcançou uma redução de $67 \%$ foi registrado 8, $9 \%$ o tratamento inicial post. Conclusões: Para todos os parâmetros clínicos: profundidade de sondagem, nível de inserção, a placa, sangramento, diferenças significativas antes e após o tratamento com as duas técnicas, gel de doxiciclina $20 \%$ e clorexidina $0,2 \%$, o sangramento mais notavelmente na redução com a utilização de doxiciclina de $67 \%$ para $8,9 \%$.

Palavras chave: Doença periodontal; doxiciclina; clorexidina; periodontopatógeno

\section{Introducción}

La enfermedad periodontal está caracterizada por ser una enfermedad multifactorial en la cual las bacterias conocidas como periodontopatógenas son el factor etiológico primario, cuya acción patógena puede verse potenciada por otros elementos perfectamente prevenibles. Esta patología afecta al periodonto de protección (Gingivitis) y al de inserción (periodontitis).En el caso de la 
periodontitis, ésta alberga millones de bacterias en la bolsa, las cuales encuentran las condiciones ideales para su multiplicación, proliferación ó propagación y cuyas asociaciones e interacciones hacen difícil su control y erradicación. Esta es una de las razones por las que se justifica usar medicación sistémica o local dentro de la bolsa periodontal como coadyuvante del tratamiento mecánico, permitiendo que aumente el porcentaje de éxito del tratamiento periodontal.

A lo largo de los años, durante la terapia periodontal se han utilizado gran variedad de antisépticos y sustancias con acción antimicrobiana en el interior de la bolsa periodontal, particularmente la desinfección de la bolsa periodontal después de la instrumentación, incrementa significativamente el porcentaje de éxito del tratamiento, de ahí que es importante el uso de una medicación dentro de la bolsa que cumpla con un efecto antimicrobiano, como es el caso del gel de doxiciclina al $20 \%$ y solución de clorhexidina al $0.2 \%$.

Resulta importante, entonces, indagar cuáles serían los efectos clínicos del uso de distintos tipos de tratamiento farmacológico (gel de doxiciclina al $20 \%$ y solución de clorhexidina al $0.2 \%$ ) como adjuntos al tratamiento mecánico en pacientes con enfermedad periodontal crónica, respuesta que se pretende dar a través del presente trabajo investigativo que tiene como objetivo: Evaluar los efectos clínicos de los tipos de tratamientos de recuperación periodontal (gel de doxiciclina al 20\% y solución de clorhexidina al $0.2 \%$ ) en pacientes con enfermedad periodontal crónica después de la terapia periodontal básica.

De esta manera se justifica, la realización de esta investigación sustentada en varios artículos científicos que avalan el uso de estos medicamentos como terapia local coadyuvante del tratamiento mecánico periodontal.

Acerca de la eficacia de un colutorio-gel acuoso de clorhexidina al 0,1\%, comparado con colutorios al $0,12 \%$ y $0,1 \%$ con alcohol evaluándolos a través del crecimiento de placa bacteriana, determinando que estas medicaciones tuvieron igual efectividad en retardar el crecimiento de placa bacteriana. (1)

La liberación de la doxiciclina al 10\% como coadyuvante en el tratamiento de periodontitis crónica en pacientes fumadores para determinar si existe reducción de bolsas periodontales, niveles de inserción, índice de placa, logrando demostrar que no existieron efectos secundarios por el uso de la 
doxiciclina, además de dar como resultados una disminución significativa en todos los parámetros analizados. (2)

Iturralde M; 2008, propone un estudio comparativo sobre la efectividad del raspado y alisado radicular combinado con aplicación de doxiciclina tópica al 10\% frente al uso del raspado y alisado solamente, en pacientes atendidos en la clínica odontológica de la Universidad San Francisco de Quito, la investigación se llevó a cabo en 30 pacientes con periodontitis crónica tanto moderada como avanzada las mismas que se dividieron en dos grupos grupo 1 al que le realizaron solo raspado y alisado mientras que al grupo 2 le realizaron raspado y alisado más la aplicación del gel de doxiciclina por un periodo de siete días la misma que la cubrieron con cemento quirúrgico para ser reevaluados a los 21 días, en este estudio se tomó en cuenta la profundidad de sondaje la misma que no demostró diferencias estadísticamente significativas para ninguno de los dos grupos, sin embargo en bolsas periodontales mayores a $7 \mathrm{~mm}$ en el grupo 2 presentaron una mayor disminución. (3)

Armas, M.; 2010, presenta un estudio de los efectos clínicos de la aplicación del gel de doxiciclina al $10 \%$ en pacientes con enfermedad periodontal crónica, realizó el estudio en dos grupos; uno que sometido exclusivamente a la terapia periodontal básica mientras que al otro grupo se añadió el gel de doxiciclina al 10\% los parámetros para ser evaluados fueron profundidad de bolsas periodontales, reducción del nivel de inserción, índice de placa y sangrado, sin que se encuentren diferencias estadísticamente significativas para los dos grupos. (4)

La presente investigación tiene como propósito comparar los efectos clínicos de la aplicación del (gel de doxiciclina al 20\%, solución de clorhexidina al $0.2 \%$ ), en pacientes con enfermedad periodontal crónica después de la terapia periodontal básica. Existe la necesidad de investigar los efectos clínicos de estos medicamentos ya que podría ser un coadyuvante de la terapia periodontal, y a su vez se pretende definir con cuál de estos medicamentos se podría obtener resultados con mayor eficacia. Si se logra observar cambios con la utilización de los mismos llegaríamos a obtener beneficios clínicos que serían reflejados en los pacientes en la consulta diaria.

Además se trata de establecer una comparación en la cual se pueda determinar si es necesaria la utilización de medicamentos o simplemente bastaría con la terapia periodontal básica, descartando la utilización de las mismas. 


\section{Materiales y métodos}

En el presente estudio experimental, se analizó a 30 pacientes con enfermedad periodontal que involucraron: 115 bolsas periodontales en las que fueron aplicadas gel de doxiciclina al 20\% y 115 bolsas periodontales en las que se aplicó clorhexidina al $0.2 \%$. Tras la colocación de la doxiciclina al 20\% se colocó cemento quirúrgico.

\section{Técnicas}

El estudio se realizó en pacientes a boca dividida en los cuales se seleccionó varios dientes con periodontitis avanzada. Se realizó raspado y alisado radicular (Figura 1), y se usó además dos tipos de sustancias en cada diente: gel de doxiciclina al 20\% y solución de clorhexidina al $0.2 \%$.La solución de clorhexidina al $0.2 \%$, se usó en forma de irrigación, mientras que el gel de doxiciclina al $20 \%$ se depositó en el sitio de la bolsa y se mantuvo por un periodo de 7 días cubierto con cemento quirúrgico.

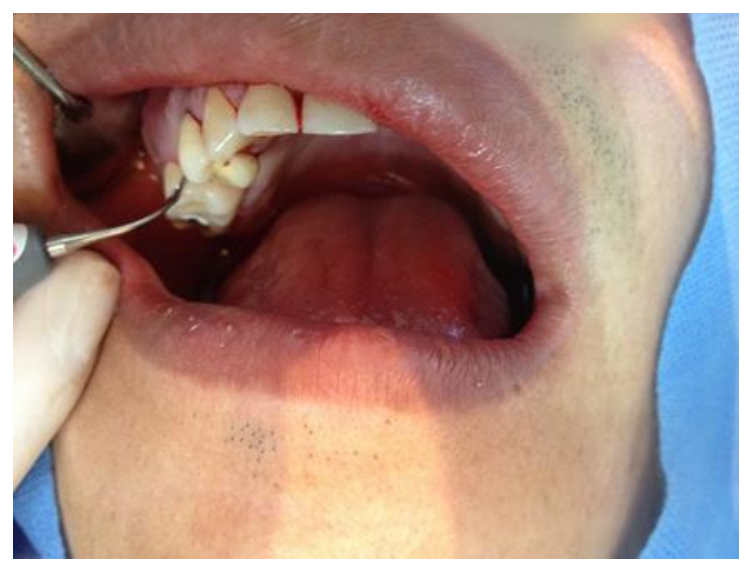

\section{Figura 1.- Raspado y alisado radicular}

Gel de doxiciclina (Figura 2) fue preparado en la ciudad de Cuenca a base de 200 cápsulas de Doxiciclina de $100 \mathrm{mg}$; el contenido de estas fue diluido en carboximetilcelulosa formando un hidrocoloide el cual fue mezclado con agua destilada para finalmente llegar a la concentración adecuada $(20 \%)$. 


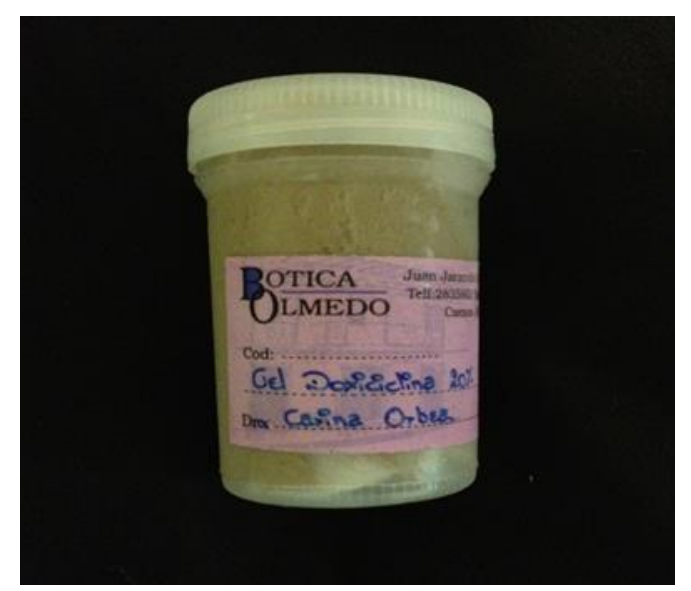

Figura 2.- Gel de Doxiciclina

\section{Medición de Profundidad de bolsa periodontal y Nivel de inserción:}

A través de una sonda periodontal (CP12), los valores recolectados son en mm.

\section{Sangrado periodontal:}

Examinación tanto clínica al momento del sondaje, como visual. (si-no).

\section{Placa dental:}

Se logra a través del conteo de superficies dentales que presentan tinción al usar liquido revelador de placa dental. Este valor se da en porcentajes.

\section{Análisis Estadístico:}

El proceso experimental en sus dos dimensiones; medición y observación permitió el registro de información en el instrumento diseñado para el efecto. Con el conjunto de datos obtenidos se elaboró una tabla de vaciado de datos con el fin de sistematizar la información en tablas y gráficas de estadística descriptiva, así como la realización de las pruebas de estadística inferencial, procesos éstos desarrollados mediante el uso del paquete estadístico SPSS versión 20 en español.

\section{Resultados}


El proceso experimental en sus dos dimensiones; medición y observación permitió el registro de información mediante una tabla de vaciado de datos con el fin de sistematizar la información en tablas y gráficas de estadística descriptiva. La prueba estadística utilizada fue t student para muestras pareadas.

Tabla 1.- Valor medio de la profundidad de sondaje antes y después de tratamiento

\begin{tabular}{|l|c|c|c|c|}
\hline \multirow{2}{*}{} & \multicolumn{2}{|c|}{ DOXICILINA } & \multicolumn{2}{c|}{ CLORHEXIDINA } \\
\cline { 2 - 5 } & INICIAL & FINAL & INICIAL & FINAL \\
\hline Media & 5,63 & 3,52 & 5,58 & 3,78 \\
\hline
\end{tabular}

Se observa que para los dos grupos la variable de profundidad de sondaje para los dos medicamentos en estudio, existe una disminución post tratamiento, diferencia que sí resultó significativa dado que $\mathrm{p}=0$.

Tabla 2.- Valor medio del nivel de inserción antes y después de tratamiento.

\begin{tabular}{|l|c|c|c|c|}
\hline \multirow{2}{*}{} & \multicolumn{2}{|c|}{ DOXICILINA } & \multicolumn{2}{c|}{ CLORHEXIDINA } \\
\cline { 2 - 5 } & INICIAL & FINAL & INICIAL & FINAL \\
\hline Media & 5,85 & 4,19 & 6,24 & 4,83 \\
\hline
\end{tabular}

En cuanto a nivel de inserción al comparar para cada medicamento el nivel inicial y final, la prueba t para muestras pareadas determinó para los dos grupos un valor $\mathrm{p}=0$, permitiendo concluir que si hay diferencias significativas en el nivel de inserción antes y después de tratamiento, no obstante la comparación del valor final del nivel de inserción para los dos tratamientos no fue de diferencia significativa $(\mathrm{p}=0,23)$. 
Tabla 3.- Valor medio del índice de placa antes y después de tratamiento

\begin{tabular}{|c|c|c|c|c|}
\hline \multirow{2}{*}{} & \multicolumn{2}{|c|}{ DOXICILINA } & \multicolumn{2}{c|}{ CLORHEXIDINA } \\
\cline { 2 - 5 } & INICIAL & FINAL & INICIAL & FINAL \\
\hline Media & 47,75 & 27,85 & 48,14 & 28,20 \\
\hline
\end{tabular}

En el índice de placa se notó una clara disminución luego del tratamiento para los dos grupos, así lo confirmó la prueba t de student para muestras pareadas, ya que en los dos casos se obtuvo $\mathrm{p}=0$.

Al comparar los valores finales del índice de placa no se encontró diferencia significativa en la acción provocada por ambos medicamentos $(\mathrm{p}=0,76)$.

En términos generales se observó una buena respuesta en cuanto a la disminución del sangrado y aparentemente se registra una mayor eficacia para la doxicilina, en la que se disminuyó de $67 \%$ inicial a solo $8,9 \%$ post tratamiento.

En el caso de esta variable al realizar un análisis comparativo de la proporción de bolsas periodontales que presentaban sangrado antes de iniciar el tratamiento se observaron diferencias significativas, de acuerdo a la prueba $\mathrm{Z}$ para la diferencia de proporciones se obtuvo un valor de $(\mathrm{p}=$ 0,003).

Los resultados anteriores han sido realizados en forma independiente, describiendo la tendencia de las magnitudes antes y después del tratamiento para cada grupo. De igual manera se pudo comprobar que el diseño experimental presenta puntos de partida similares para las variables cuantitativas en cuestión, no así para la presencia de sangrado (cualitativa).

Tabla 4.- Variación de profundidad de sondaje, nivel de inserción e índice de placa.

\begin{tabular}{|c|c|c|c|c|}
\hline MEDICAMENTO & $\begin{array}{c}\text { PROFUNDIDAD DE } \\
\text { SONDAJE (MM) }\end{array}$ & $\begin{array}{c}\text { NIVEL DE } \\
\text { INSERCIÓN } \\
\text { (MM) }\end{array}$ & $\begin{array}{c}\text { ÍNDICE DE } \\
\text { PLACA \% }\end{array}$ & SANGRADO\% \\
\hline DOXICILINA & 2,12 & 1,66 & 19,9 & 58,1 \\
\hline CLORHEXIDINA & 1,8 & 1,4 & 19,9 & 47,7 \\
\hline Significancia (P) & $\mathrm{P}=0$ & $\mathrm{P}=0,08$ & $\mathrm{P}=0,98$ & $\mathrm{P}=0,04$ \\
\hline
\end{tabular}


Se observó que la doxicilina demuestra valores de variación más altos para las cuatro magnitudes ensayadas, encontrándose diferencias significativas en el nivel de la profundidad de sondaje y la proporción de presencia de sangrado $(\mathrm{p}<0,05)$.

\section{Discusión}

Es de gran conocimiento que la presencia de microorganismos juega un papel fundamental en el desarrollo de la enfermedad periodontal; ante lo cual se han llevado a cabo varios estudios empleando ciertos medicamentos en el interior de las bolsas periodontales con el objetivo de disminuir o erradicar la carga bacteriana, considerando que uno de los objetivos del tratamiento periodontal es precisamente la eliminación de los microorganismos periodontopatógenos existentes en las bolsas periodontales, evitando además una posible reinfección.

La terapia mecánica reduce los microorganismos existentes en la bolsa periodontal pero no llegan a ser completamente eliminados de su interior, de hecho Machion, L. et al; 2006, (2) en su estudio realizado durante dos años, demuestra que el grupo en el que aplicó el raspado y alisado junto con la doxiciclina al 10\%, presentó una disminución especialmente en bolsas profundas ya que a los 45 días del tratamiento este indicó una reducción de $1.63 \pm 1.16 \mathrm{~mm}$ versus $1.50 \pm 0.55 \mathrm{~mm}$ al grupo que realizaron solo el raspado y alisado.

Aimetti, M. et al, 2004, (5) evaluó las características clínicas, radiológicas y microbiológicas en lesiones periodontales persistentes después de la administración de la tetraciclina de forma local (Actisite) y solo con raspado radicular, determinó que al analizar los controles a los seis y doce meses no existieron diferencias significativas en el índice de placa, en cuanto a la profundidad de sondaje y ganancia de inserción, existió una mejoría, radiográficamente el nivel óseo mostró un aumento significativo en el grupo prueba, y microbiológicamente pudieron observar que fue más eficaz el tratamiento combinado ya que eliminó a las bacterias periodontopatógenas existentes en dichas bolsas. Se concluyó que la utilización de antibiótico de manera local logró una disminución de la profundidad de sondaje y la eliminación de bacterias periodontopatógenas que son las causantes de la enfermedad periodontal.

La efectividad del raspado y alisado radicular sin y con la aplicación del gel de doxiciclina al 10\%, (3) al igual que Armas M; 2010. (4) no muestran en sus estudios diferencias estadísticamente 
significativas en ninguno de los dos grupos, sin embargo Iturralde hace referencia que en bolsas periodontales mayores a $7 \mathrm{~mm}$ en el grupo tratado con doxiciclina $10 \%$ se presentó una mayor disminución.

Sin embargo Machion, L. et al 2006 (2) al igual que Iturralde 2008, (3); hacen referencia que en las bolsas periodontales con mayor profundidad de sondaje $(7 \mathrm{~mm})$ se encuentra una mayor reducción al colocar tetraciclina y tetraciclina semisintética respectivamente que en los grupos control.

Arweiler NB, \& col. 2006; (6) demostró en cambio que la solución de clorhexidina convencional resultó ser el producto con el mayor efecto de inhibición de la placa y antibacteriano en comparación con la solución de placebo, pero al ser comparada el efecto del colutorio de clorhexidina convencional con el colutorio de clorhexidina más la sustancia para evitar las manchas (ADS) esta fue incapaz de igualar la inhibición de la placa que presentó la solución de clorhexidina convencional. Determinando así que el uso del enjuague bucal convencional a base de clorhexidina sigue siendo muy efectivo en la inhibición bacteriana. Así lo confirman autores como Trejo S; 2010, (7) quien considera que la clorhexidina es un compuesto quimioterapéutico líder en el control de la placa bacteriana.

En el artículo presentado por Arweiler, NB; 2006, (6) indica que el digluconato de clorhexidina es la sustancia antimicrobiana más eficaz en prevenir o reducir la formación de placa dental, ya que el ingrediente activo ha demostrado ser el agente más eficaz para reducir la placa y la gingivitis esto concuerda con lo que menciona Choe, JY; 2012, (8) quien mantiene que la clorhexidina presenta un efecto bactericida de acción rápida contra un alto rango de bacterias tanto gram - positivas y negativas, así como los hongos, de igual manera Zvonimir, C; 2006; Texeira, AL; 2007; Barrios G; 2004, menciona que esta sustancia presenta una mayor eficacia en la inhibición de la placa, ofreciendo un amplio espectro antibacteriano, efectivo contra microorganismos anaerobios, aerobios, gramnegativos y grampositivos. $(9,10,11)$

Después de comparar los resultados obtenidos en éste estudio y los resultados de los diferentes artículos revisados se puede indicar que pese a que no hay evidencias absolutas de la efectividad de los dos medicamentos ensayados, éstos si presentan efectos clínico efectivos en el tratamiento de la enfermedad periodontal, dando como resultado una mejor acción al gel de doxiciclina $20 \%$ la misma que debido a su concentración pudo influir en los resultados. 


\section{Conclusiones}

Existe una gran variedad de microorganismo periodontopatógenos identificados dentro de la bolsa periodontal identificados como agentes etiológicos de las enfermedades periodontales.

Tanto la clorhexidina como la doxiciclina pueden ser consideradas como alternativas válidas para eliminar bacterias encontradas en el interior de la bolsa periodontal, por lo que se concluye que el uso de este antiséptico y antibiótico es idóneo en el caso de pacientes con enfermedad periodontal.

La acción antimicrobiana demostrada de la doxiciclina se debe a que ésta es un antibiótico perteneciente al grupo de las tetraciclinas semisintéticas, que son antibióticos de amplio espectro actuando contra bacterias gram positivas y gram negativas.

Los dos medicamentos son efectivos para la disminución de la profundidad de sondaje, el nivel de inserción, disminución de sangrado, y la disminución del índice de placa, pero como pudimos ver en este estudio el gel de doxiciclina al $20 \%$ tuvo mejores resultados por lo que se puede concluir que esta tetraciclina semisintética es efectiva como coadyuvante en el tratamiento de la enfermedad periodontal.

\section{Bibliografía}

1. Yévenes I, Norero H, Reyes J, Rivera S. Efecto comparativo de nuevo colutorio-gel de clorhexidina con colutorios comerciales en el crecimiento de placa en 24 horas. Avances en periodoncia e implantología oral. 2006 dic; 18(3).

2. Machion L, Andia D, Lecio G, Nociti FJ, Casati M, Sallum A, et al. Locally delivered doxycycline as an adjunctive therapy to scaling and root planing in the treatment of smokers: a 2-year follow-up. J Periodontol. 2006 apr; 77(4): p. 606-613.

3. Iturralde-Piedra MJ. Estudio comparativo sobre la actividad del raspado y alisado radicular combinado con aplicación de doxiciclina tópica al 10\% frente al uso de raspado y alisado radicular solamente, en pacientes atendidos en la clínica odontológica de la USFQ. Tesis -

Postgrado Odontología. Quito: Universidad San Francisco de Quito, Especialista en Periodoncia; 2008. Report No.: http://repositorio.usfq.edu.ec/handle/23000/529. 
4. Armas ME. Evaluación de los efectos clínicos de la aplicación de gel de doxiciclina al 10\% en el tratamiento de la periodontitis crónica. Informe Final. Quito: Universidad Central del Ecuador, Postgrado Periodoncia; 2010.

5. Aimetti M, Romano F, Torta L, Cirillo D, Caposio P, Romagnoli R. Debridement and local application of tetracycline-loaded fibres in the management of persistent periodontitis: results after 12 months. J Clin Periodontol. 2004 mar; 31(3): p. 166-172.

6. Arweiler N, Boehnke N, Sculean A, Hellwig G, Auschill T. Differences in efficacy of two commercial $0.2 \%$ chlorhexidine mouthrinse solutions: a 4-day plaque re-growth study. $\mathrm{J}$ Clin Periodontol. 2006 may; 35(5): p. 334-339.

7. Trejo S. Fundamentos de farmacología: Trillas; 2010.

8. $\quad$ Choe J. Drug actions and Interactions. New York: Amolca; 2012.

9. Zvonimir C, Saxer UP. Utilización de antisépticos en la prevención y el tratamiento de la periodontitis. Quintessence: Publicación internacional de odontología. 2005; 18(5): p. 249258.

10. Teixeria-Meira L, Correia-Todescan M, Azouble E, Bittencourt S, Fonseca-Azouble MC. Uso de antimicrobianos locais em periodontia: Uma Obordagem crítica. Periodontia. 2007 mar; 17(1).

11. Barrios GG, Caffesse R, Jiménez M, Manton S, Polson A, Sendyk W. Odontología. Bogotá: Editar; 2004.

12. SEPA, Echevarría JJ, Carrión JB. Manual SEPA de Periodoncia y Terapéutica de Implantes: Fundamentos y guía práctica. Madrid: Medica Panamericana; 2005.

13. Bruschi ML, Panzeri H, De Freitas, Guimarães Lara EH, Daflon-Gremião P. Sistemas de liberação de fármaco intrabolsa periodontal. Revista Brasileira de Ciências Farmacêuticas. 2006 mar; 42(1).

14. Newman MG, Takei H, Klokecold P. Periodontología Clínica de Carranza. 11th ed. Carranza F, editor. New York: Amolca; 2014. 
15. Heitz-Mayfield L. How effective is surgical therapy compared with nonsurgical debridement?. Periodontol 2000. 2005; 37(1).

16. Rioboo CM, Bascones A. Factores de riesgo de la enfermedad periodontal: factores genéticos. Avances en Periodoncia. 2005; 17(2): p. 69-77.

17. Guilarte C, Perrone M. Microorganismos de la placa dental relacionados con la etiología de la periodontitis. Acta Odontológica Venezolana. 2004; 42(3).

18. Feng Z, Weinberg A. Role of bacteria in health and disease of periodontal tissues. Periodontol 2000. 2006; 40(1): p. 50-76.

19. Walker C, Karpinia K, Baehni P. Chemotherapeutics: antibiotics and other antimicrobials. Periodontol 2000. 2004; 36(1): p. 146-165.

20. Botero J, Bedoya E. Determinantes del diagnóstico periodontal. Revista clínica de periodoncia, implantología y rehabilitación oral. 2010 agos; 3(2).

21. Lindhe J, Niklaus P, Thorkil K. Periodontología Clínica e Implantología Odontoógica. 5th ed. Buenos Aires: Panamericana; 2009.

22. Bascones M, Mudarra M, Perea P. Antisépticos en el tratamiento de la enfermedad periodontal. Avances en Periodoncia e Implantología Oral. 2002 oct; 14(3). 